\title{
Zu diesem Heft - Die Stunde der Wissenschaft?
}

Selten waren Wissenschaftlerinnen und Wissenschaftler öffentlich so präsent und einflussreich wie in den vergangenen Monaten, in denen sich das Corona-Virus ausgehend von China und Europa in rasanter Geschwindigkeit über die ganze Welt ausgebreitet hat. Anfangs waren es vor allem Virologen und Epidemiologen, die aufgrund ihrer Expertise in die engsten Beraterzirkel der Bundesregierung aufgenommen wurden. Die tiefgreifenden Umwälzungen infolge dieser Krise, die bislang nur erahnt werden können, zeigen allerdings, dass es bei den Maßnahmen, die zu ihrer Bewältigung ergriffen werden, nicht allein um das "nackte Leben " gehen kann, sondern dass auch die Gesellschaft als Ganze im Mittelpunkt der Forschung stehen muss. Hier ist die gesamte Breite der Sozial- und Geisteswissenschaften gefordert. Ihre Aufgabe ist es nicht nur, die aktuellen Prozesse, Deutungen und Maßnahmen in verschiedenen Ländern auf ihre nicht-virologischen Aspekte hin kritisch zu befragen, sondern auch die mit der Krise verbundenen wirtschaftlichen, sozialen, politischen, kulturellen und (sozial)psychologischen Auswirkungen umfassend zu erforschen und auf diese Weise Orientierungswissen für den politischen Gestaltungsprozess zur Verfügung zu stellen. Dieser Herausforderung wird sich auch der Leviathan annehmen, einen ersten Schwerpunkt zum Thema gibt es im nächsten Heft.

Um die unmittelbaren ökonomischen Verheerungen, die mit den Ausgangsbeschränkungen und Kontaktverboten verbunden sind, abzufedern, hat die deutsche Regierung - hier werden Erinnerungen an die letzte Finanzkrise wach - milliardenschwere Konjunktur- und Rettungspakete geschnürt und ein vorläufiges Ende der lange als alternativlos propagierten »Schwarzen Null« angekündigt. Um die Finanzmärkte "zu beruhigen ", hat die Europäische Zentralbank ein Notfallprogramm aufgesetzt, das eine massive Ausweitung der Anleiheankäufe vorsieht. Damit dürfte die Hoffnung all jener, die sich ein baldiges Ende von Niedrigzins und quantitative easing herbeiwünschten, erst einmal begraben sein (wenngleich sie sich zumindest durch das im Mai gefällte Urteil des Bundesverfassungsgerichts bestätigt fühlen dürften). Für unsere Autoren Jan Sparsam und Malte Flachmeyer ist das jedoch kein Grund zur Beunruhigung. In ihrem Debattenbeitrag argumentieren sie, dass die Sehnsucht nach einer Rückkehr zur geldpolitischen "Normalität « nicht nur auf der zweifelhaften Annahme basiere, dass es vor der Finanz- und Staatsschuldenkrise so etwas wie eine neutrale und in diesem Sinne auch unpolitische Geldpolitik gegeben habe, sondern darüber hinaus auch die Komplexität der Problemlagen innerhalb der EU verkenne. Geldpolitik müsse vielmehr »als Resultat struktureller, über einen längeren Zeitraum wirtschafts- und fiskalpolitisch verursachter Probleme " angesehen werden, »denen die Sehnsucht nach Normalität analytisch und politisch nicht gerecht « werde.

Blickt man ein Stück weiter zurück in die Geschichte, wird schnell deutlich, dass die Niedrigzinspolitik nicht nur als eine Folge, sondern auch als eine Entstehungsbedingung der Finanzkrise von 2008 zu begreifen ist. Die durch die US-Zentral- 
bank ausgelöste Leitzinsabsenkung nach 9/11 hat im Zusammenspiel mit der von der Clinton-Regierung forcierten »Eigenheim für alle«-Politik einen schuldenfinanzierten Immobilienboom ausgelöst, der letztlich zum Crash führte und nicht nur amerikanische Banken, sondern das Finanzsystem weltweit in den Abgrund riss. In Deutschland gab es vor der Finanzkrise keinen vergleichbaren Immobilienboom. Hierzulande ist erst im vergangenen Jahrzehnt ein rasanter Anstieg von Hauspreisen und Mieten zu beobachten, der gesellschaftliche Sprengkraft birgt und das Wohnen als soziale Frage wieder verstärkt auf die politische Agenda rückt. Till Baldenius, Sebastian Kohl und Moritz Schularick legen in ihrem Debattenbeitrag erstmals Zahlen zu den Verteilungswirkungen dieses Booms vor. Sie zeigen anhand eigener Berechnungen, dass deutsche Immobilienbesitzer*innen in den letzten Jahren um knapp drei Billionen Euro reicher geworden sind, wobei allein die Hälfte dieses Vermögens den reichsten zehn Prozent zufiel. Die untere Hälfte der Haushalte ist den Autoren zufolge nicht nur fast leer ausgegangen, hinzu komme, dass - relativ gesehen - gerade für die unteren Einkommensgruppen die Mieten besonders stark angestiegen seien. Im Schnitt gäben die unteren 20 Prozent der Haushalte mittlerweile knapp 40 Prozent ihres Einkommens für Wohnen aus, während sich dieser Anteil bei den oberen 20 Prozent sogar leicht reduziert habe und heute nur etwa 15 Prozent betrage. Vor diesem Hintergrund ist es nicht überraschend, dass die Einnahmeausfälle infolge der Corona-Pandemie viele Mieter*innen in Bedrängnis bringen. Schon zu Beginn der Krise war absehbar, dass es für viele Haushalte nicht mehr möglich sein wird, ihre Mieten zu bezahlen. Die Bundesregierung beschloss daraufhin ein Gesetz, demzufolge Mietschulden aus den Monaten April bis Juni kein Kündigungsgrund sein dürfen, allerdings nur unter der Bedingung, dass die Rückstände innerhalb von 24 Monaten beglichen werden.

Der Anstieg der Einkommens- und Vermögensungleichheit in den letzten Jahrzehnten wurde auf städtischer Ebene von Gentrifizierungs- und Segregrationsprozessen begleitet, die zunehmend homogene, nach Einkommen und Bildung getrennte Wohnviertel hervorbringen. Wo in den Nachkriegsjahrzehnten die Lösung des Wohnungsproblems in erster Linie in der öffentlichen Förderung des Wohnungsbaus bestand, stehen in Zeiten der neoliberalen Umgestaltung von Stadtgesellschaften andere Mittel zur Verfügung. Eines davon ist die kommunale Sozialplanung mit ihrem zentralen Instrument der Sozialberichterstattung, denen Markus Baum und Marius Otto in ihrem Beitrag allerdings paradoxale Effekte unterstellen. Diese Art von Sozialplanung begünstige durch ihren Fokus auf effiziente Ressourcenallokation und die Identifikation von »Problemvierteln « die territoriale Stigmatisierung entsprechender Orte, womit sie entgegen ihrer Absicht die strukturelle Segregation marginalisierter Gruppen noch verstärke. Die Autoren zeigen Perspektiven einer reflexiven Sozialplanung auf, deren Anspruch es sei, diesen Problemen bewusst entgegenzusteuern.

Mit der Corona-Krise nehmen nicht nur die ökonomischen Probleme zu, vielerorts wird nun auch die Frage gestellt, welchen Einfluss die aktuellen Entwicklungen auf die politischen Kräfteverhältnisse in Deutschland und darüber hinaus haben werden. Werden Rechtspopulismus und Rechtsextremismus gestärkt aus 
der Krise hervorgehen? Viele Expert*innen vermuten, dass das Autoritäre in der Krise zunehmen wird. ${ }^{1}$ Für verlässliche Befunde ist es noch zu früh, zu beobachten ist allerdings bereits jetzt, dass in der gegenwärtigen Situation eines gefühlten Kontrollverlusts Verschwörungstheorien einen regelrechten Boom erleben. Selbst die Bundesregierung befürchtet, dass die Gefahr rechtsextremer Anschläge steigen könnte, weshalb die Opposition eine besonders strenge Überwachung rechter Netzwerke fordert. Ein Blick auf die neurechte Webseite sezession.de zeigt, dass sich auch im rechtsintellektuellen Milieu eine gewisse Aufbruchstimmung breitmacht. Die Auswirkungen der Corona-Pandemie scheinen den nationalistischen Traum von einer Welt zu erfüllen, in der Globalisierung und Kosmopolitismus der Vergangenheit angehören. Der Autor Florian Sander schreibt: »Das öffentliche Leben im hyperschnell gewordenen, globalisierten und entgrenzten Spätkapitalismus wird mit einem Mal urplötzlich entschleunigt: Wir werden gezwungen zum Innehalten, zur Besinnung, zum In-uns-gehen." Diese Entschleunigung, so die Hoffnung, soll zu einer allgemeinen historischen Rückwärtsbewegung führen: »Wir erleben ein neues gesellschaftliches Miteinander, das nicht nur die Rückkehr des Nationalstaates, sondern sogar die Rückkehr des Volkes (anstatt: der >Bevölkerung<) bedeuten kann [...]. $«^{2}$ Zur Formulierung und Rechtfertigung ihrer nationalistisch-völkischen Ideologien beruft sich die Neue Rechte auf verschiedene Denktraditionen, die allerdings nicht immer dem rechten Spektrum zuzuordnen sind. Das zeigt Carolin Amlinger in ihrem Essay, der sich mit der selektiven Aneignung des postmodernen Denkens durch die Neue Rechte befasst. Die Autorin zeichnet darin nach, wie sich rechte Denker*innen den Gestus einer radikalen Modernekritik einverleiben und für ihre eigenen Ziele fruchtbar machen.

Mit dem Gegenpart des Linksintellektuellen beschäftigt sich Alfons Söllner in seinem Essay über Theodor W. Adorno. Ausgehend von seinen Schriften der frühen Bundesrepublik (inklusive des 2019 erschienenen Nachlassbands der unveröffentlichten Vorträge) geht er der Frage nach, inwiefern dieser als " political scholar « zu begreifen sei. Adornos Vortrag »Aspekte des neuen Rechtsradikalismus ", den er 1967 im Kontext der erstarkenden NPD und ihres Einzugs in mehrere Länderparlamente in Wien hielt und der ebenfalls im Nachlassband erschien, hat im letzten Jahr eine besondere mediale Aufmerksamkeit erfahren. Viele Kommentator*innen meinten, in dem Text eine Erklärung für den jüngsten Aufstieg rechtspopulistischer Parteien zu finden. Wenngleich Söllner selbst zur Vorsicht mahnt, »einem sozialphilosophischen Klassiker $[$ [...] das autoritative Urteil über eine gründlich veränderte Gegenwart « aufzubürden, und mutmaßt, dass Adorno selbst »dergleichen Genieästhetik zumindest für das profane Feld der Tagespolitik abgelehnt" hätte, scheint der Text dennoch einen wunden Punkt zu treffen. Das

1 Siehe www.zeit.de/gesellschaft/zeitgeschehen/2020-04/wilhelm-heitmeyer-coronavirus-ve rschwoerungstheorien-finanzmarkt-rechtsradikalismus; www.tagesspiegel.de/politik/diedunkle-seite-des-wir-gefuehls-trotz-aller-solidaritaet-in-der-pandemie-droht-das-autorita ere/25754430.html (Zugriffe vom 21.04.2020).

2 Siehe https://sezession.de/62398/ausnahmezustand-die-rueckkehr-des-politischen (Zugriff vom 20.04.2020). 
erschließt sich besonders dort, wo Adorno die ökonomischen Voraussetzungen des Rechtsradikalismus benennt: die »Konzentrationstendenz des Kapitals « und die auseinanderdriftende Schere zwischen Arm und Reich, die zu Deklassierung und Abstiegsängsten in der Bevölkerung führe und damit Radikalisierung begünstige. Hier sind durchaus Parallelen zu den aktuellen Ursachen des erstarkenden Rechtspopulismus zu beobachten. Heute kommt allerdings eine neue gesellschaftliche Konfliktlinie hinzu, die auf kulturellen Faktoren basiert und von der in den 1960er Jahren noch nicht die Rede sein konnte: Einschlägigen Untersuchungen zufolge kam es in den vergangenen Jahrzehnten zu einer zunehmenden Polarisierung zwischen Globalisierungsgewinner*innen und Globalisierungsverlierer*innen, zwischen Kosmopolit*innen und Kommunitarist*innen, der für den Erfolg des Rechtspopulismus eine wesentliche Bedeutung zugeschrieben wird.

Im letzten Leviathan hat sich um beide Konfliktlinien, die ökonomische und die kulturelle, eine Debatte entspannt, die nicht nur die Frage nach dem Aufstieg des Rechtspopulismus, sondern auch die nach der Zukunft der Sozialdemokratie berührt. Michael Zürn, der an der Erforschung der kulturellen Konfliktlinie maßgeblich beteiligt war und auf den sich daher einige Autoren aus dem letzten Heft des Leviathan implizit oder explizit berufen, hat sich nun eingemischt. In seinem Kommentar nimmt er zum einen Klarstellungen vor, was die Konzeptionalisierung der Konfliktlinie zwischen Kosmopoliten und Kommunitaristen betrifft (Floris Biskamp hatte deren empirische Existenz in seinem Beitrag angezweifelt), zum anderen führt er den Autoren Michael Hartmann, Floris Biskamp und Carsten Nickel die Grenzen vor Augen, an die deren Analysen aus seiner Sicht stoßen. Er argumentiert, dass die Unterscheidung von ökonomischen und kulturellen Faktoren zwar richtig sei, dabei aber ein dritter Aspekt, die sozio-politische Dimension, aus dem Blick gerate. Die Chance der SPD sieht er weder in einer Rückbesinnung auf den Traditionalismus (Nickel) noch in der ökologischen Neuerfindung einer linken Sozialdemokratie (Biskamp), sondern in einem verstärkten Internationalismus, der die Ausschlüsse einer nationalstaatlich eingehegten Sozialdemokratie überwindet.

Um die Ausbreitung des Corona-Virus einzudämmen, denken Politik und Behörden seit längerem darüber nach, die Mobilfunkdaten der Bürger*innen zu nutzen, um die Kontaktpersonen von Infizierten besser identifizieren und isolieren zu können. In der Bevölkerung und insbesondere unter Datenschützer*innen wird diese Forderung nicht ohne Bedenken wahrgenommen. In die Debatte hat sich auch der Whistleblower Edward Snowden eingeschaltet, um vor einer Ausbreitung staatlicher Überwachungsmaßnahmen und der damit verbundenen weiteren Einschränkung der zivilen, öffentlichen und individuellen Freiheitsrechte zu warnen. Der ehemalige CIA-Mitarbeiter polarisiert die öffentliche Meinung wie kaum eine andere Figur. Durch seine Enthüllungen der Überwachungs- und Spionageaktivitäten von Geheimdiensten ist er zur Ikone von Datenschützer*innen und Menschenrechtsaktivist*innen weltweit geworden. Für sie ist er Held und Märtyrer, der im Kampf für die Freiheit noch dazu bereit ist, sein eigenes Leben zu opfern. Für die anderen gilt er als gefährlicher Staatsfeind, der sich zu Recht wegen Hochverrat vor Gericht verantworten muss. Doch was bedeutet es eigentlich auf einer 
theoretischen Ebene, ein Verräter zu sein? Und welchen Stellenwert hat der Verrat in demokratischen Gesellschaften? Diesen Fragen geht Martin Oppelt in seinem Aufsatz aus einer radikaldemokratischen Perspektive nach. In Auseinandersetzung mit einer konservativen, herrschaftskritischen und liberalen Lesart entwickelt er eine demokratietheoretische Konzeption von Verrat, die ihm das Potenzial zuschreibt, »den immer nur temporär stillgelegten Streit um die politische Ordnung und die kollektive Identität des Gemeinwesens « zu entfachen und durch sein "eröffnendes" oder "aufdeckendes Nein « die bestehende Ordnung der Dinge infrage zu stellen.

Mit einer anderen, milderen Form des politischen Protests befassen sich Angela Graf, Maria Keil und Peter Ullrich in ihrem Aufsatz über die Situation des akademischen Mittelbaus in Deutschland - oder genauer: mit dem deutlicher wahrnehmbaren Ausbleiben von Protest. Zum einen gehen sie mit Hirschman und Bourdieu der Frage nach, warum sich angesichts der hohen Unzufriedenheit mit den prekären Beschäftigungsverhältnissen bislang relativ wenig Widerstand formiert hat. Zum anderen greifen sie auf die Erkenntnisse der Protest- und Bewegungsforschung zurück, um die Bedingungen erfolgreicher Mobilisierung auf das Feld des akademischen Mittelbaus anzuwenden. Dabei wird deutlich, dass dem durchaus wachsenden Problembewusstsein in der (Fach-)Öffentlichkeit eine Reihe von strukturellen und kulturellen Faktoren gegenübersteht, die die Artikulation von Widerspruch hemmt. Ob der aktuelle Strukturwandel des Hochschulsystems eine Verbesserung der Situation bewirken könnte, wird sich den Autor"innen zufolge erst noch zeigen müssen.

Um einen der größten Proteste der jüngeren Vergangenheit ist es seit dem Beginn der Corona-Pandemie seltsam still geworden. Die aktuellen Ereignisse, so scheint es, haben der Klimabewegung ihre wichtigsten Grundlagen entzogen. Der renommierte Klimaforscher Hans Joachim Schellnhuber hofft in einem Interview mit der Frankfurter Allgemeinen Zeitung dennoch, dass die Gesellschaft und insbesondere die politischen Entscheidungsträger*innen aus der Krise lernen und der Physik eines Tages so sehr vertrauen werden wie derzeit der Virologie. Seine Hoffnung basiert auf der Beobachtung, dass sich wesentliche Charakteristika der Corona-Krise auch auf die globale Klimakrise übertragen lassen: »Die unerbittliche Gültigkeit der Naturgesetze; die kritische Bedeutung der Rechtzeitigkeit; die gelegentliche Notwendigkeit, alle Waffen, die man besitzt, ins Feld zu führen; die Bereitschaft, das Leben über das Geld zu stellen. Und auch die ominöse Kurve der weltweit kumulierten Covid-19-Fälle hat ein ikonisches Gegenstück, nämlich die berühmte Mauna-Loa-Kurve des Anstiegs der atmosphärischen CO2-Konzentration. $\aleph^{3}$ Schellnhuber ist sich der Unterschiede zwischen Corona- und Klima-Krise jedoch nicht weniger bewusst: hier die unmittelbare Virus-Bedrohung, dort die erst in Zukunft spürbaren Auswirkungen des Klimawandels; hier die absehbare Befristung des Ausnahmezustands, dort die Notwendigkeit eines dauerhaften Verzichts. Es gibt im Moment wenig Grund zur Annahme, dass die Regierungen die-

3 Hans Joachim Schellnhuber 2020. „Die Seuche im Anthropozän «, in Frankfurter Allgemeine Zeitung vom 16. April 2020, S. 9. 
ser Welt eines Tages, wenn das Virus unter Kontrolle gebracht wurde, ähnlich entschlossen handeln werden, um die Menschen vor den nicht minder tödlichen Auswirkungen der ökologischen Krise zu retten. Vieles wird wahrscheinlich auch davon abhängen, ob in der Klima-Krise vielleicht doch noch die Stunde der Wissenschaft schlagen wird.

Claudia Czingon 